\title{
Lateral straggling of implanted aluminum in 4H-SiC (i)
}

Cite as: Appl. Phys. Lett. 116, 012101 (2020); https://doi.org/10.1063/1.5132616

Submitted: 18 October 2019 . Accepted: 13 December 2019. Published Online: 02 January 2020

(D) J. Müting, V. Bobal, T. Neset Sky, L. Vines, and (D) U. Grossner

\section{COLLECTIONS}

EP This paper was selected as an Editor's Pick
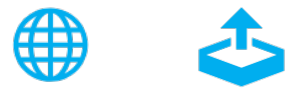

\section{ARTICLES YOU MAY BE INTERESTED IN}

Monolayer GaN excitonic deep ultraviolet light emitting diodes

Applied Physics Letters 116, 013101 (2020); https://doi.org/10.1063/1.5124828

Field driven recovery of the collective spin dynamics of the chiral soliton lattice

Applied Physics Letters 116, 012403 (2020); https://doi.org/10.1063/1.5131067

Development of $4 \mathrm{H}-\mathrm{SiC}$ Schottky np diode with high blocking voltage and ultralow onresistance

Applied Physics Letters 116, 012103 (2020); https://doi.org/10.1063/1.5130732

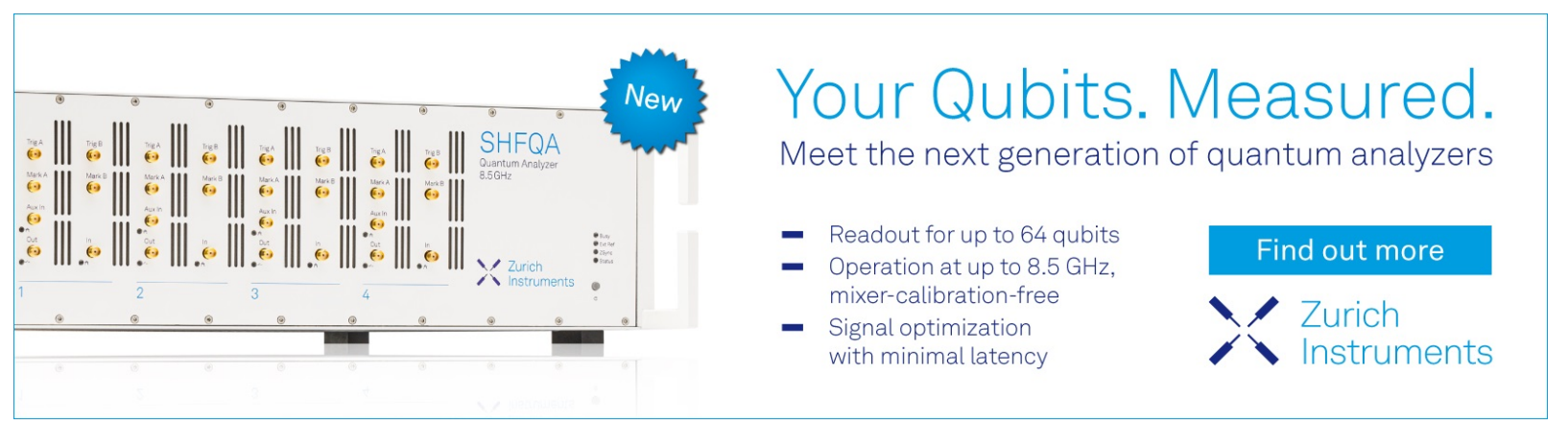




\title{
Lateral straggling of implanted aluminum in $4 \mathrm{H}-\mathrm{SiC}$ (EP
}

Cite as: Appl. Phys. Lett. 116, 012101 (2020); doi: 10.1063/1.5132616

Submitted: 18 October 2019 . Accepted: 13 December 2019 .

Published Online: 2 January 2020

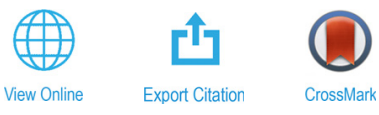

J. Müting, ${ }^{1, a)}$ (D) V. Bobal, ${ }^{2}$ T. Neset Sky, ${ }^{2}$ L. Vines, $^{2}$ and U. Grossner ${ }^{1}$ (D)

\author{
AFFILIATIONS \\ ${ }^{7}$ Advanced Power Semiconductor Laboratory, ETH Zurich, 8092 Zurich, Switzerland \\ ${ }^{2}$ Department of Physics, Center for Material Science and Nanotechnology, University of Oslo, O316 Oslo, Norway

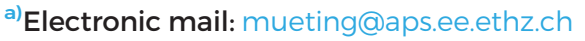

\begin{abstract}
The implantation of aluminum into $4 \mathrm{H}-\mathrm{SiC}$ is studied using secondary ion mass spectrometry. In particular, two-dimensional concentration profiles are obtained, which allow the investigation of lateral straggling and its dependence on the crystallographic orientation. A high dose, medium energy aluminum implantation is studied in great detail. It shows an asymmetric distribution due to the $4^{\circ}$-off axis growth of the epitaxial layer. The lateral straggling is found to be in the range of several micrometers for a concentration of $1 \times 10^{15} \mathrm{~cm}^{-3}$, which is contrary to the expectation given by most simulation studies. Implantations performed at different orientations support the idea that lateral straggling highly depends on the particular channeling opening.
\end{abstract}

Published by AIP Publishing. https://doi.org/10.1063/1.5132616

Silicon carbide ( $\mathrm{SiC}$ ) offers a large bandgap, a high critical electric field, and a high thermal conductivity, which make it an ideal candidate for high power electronic applications. ${ }^{1,2}$ To create semiconductor devices based on $\mathrm{SiC}$, ion implantation is used to introduce dopants into the crystal, since diffusion-based processes are not feasible. ${ }^{3}$ In the case of a metal-oxide-semiconductor field-effect transistor (MOSFET), ion implantation is used to form the p-body and the $\mathrm{n}^{+}$-source, which form the channel region of the MOSFET. The channel is controlled by the gate to turn on and off the device. In order to obtain a specific threshold voltage and a predictable channel behavior, doping regions need to be precise in terms of geometry and concentration. During ion implantation, donors or acceptors are accelerated toward the semiconductor, and by choosing the dose and energy of the ions, a specific depth profile of the dopant is obtained. However, the implantation profile may be altered if the ions enter one of the crystallographic directions, known as channeling. Vertical channeling in $\mathrm{SiC}$ is known to highly affect the resulting implantation profile depending on the particular channel direction. ${ }^{4}$ Lateral straggling is equally important, especially for lateral geometries such as the channel region of the MOSFET, ${ }^{5}$ but has not been experimentally studied that extensively. There exist several simulation studies based on Monte Carlo codes that estimate lateral straggling of aluminum in SiC. The results range from around $500 \mathrm{~nm}$ up to several micrometers. ${ }^{6-9}$ Experimentally determining lateral straggling in $\mathrm{SiC}$, however, could not be achieved yet. ${ }^{10}$ Hence, the aim of this paper is to use two-dimensional secondary ion mass spectrometry (SIMS) to evaluate lateral straggling of aluminum in $4 \mathrm{H}-\mathrm{SiC}$ and its dependence on the crystallographic structure.

In order to resolve a three-dimensional implantation, a high spatial resolution in all directions is required. One approach is spreading resistance profiling, which has been used to detect lateral straggling in silicon and $\mathrm{SiC}^{10,11}$ It has a high lateral resolution of some $10 \mathrm{~nm}$. However, it can only determine the concentration on a surface, i.e., the sample requires intense modification prior to measuring. In the case of $\mathrm{SiC}$, additional problems occur regarding the contact formation between the probe and the semiconductor. SIMS, on the other hand, uses a focused ion beam to sputter a target and analyze the hereby generated secondary ions. As a result, SIMS has a high degree of freedom and is thus the most common technique to measure vertical implantation profiles. These depth profiles mainly require a high vertical resolution. For three-dimensional measurements, also the lateral resolution, which depends on the diameter of the primary beam, is of great importance. In this work, a lateral resolution of $2 \mu \mathrm{m}$, a vertical resolution of $5-10 \mathrm{~nm}$, and a sensitivity of five decades for aluminum were established, exhibiting a good compromise for the various flexible parameters in SIMS. The spatial resolution is confirmed by measuring a reference sample, where silicon stripes separated by $2 \mu \mathrm{m}$ can be clearly distinguished with the given beam. Additionally, the beam diameter of around $2 \mu \mathrm{m}$ is verified by investigating the slope of the sputtered crater. 
A $4 \mathrm{H}$-SiC wafer with a $17 \mu \mathrm{m}$ thick, $4^{\circ}$-off axis grown, $\mathrm{n}$-type epitaxial layer $\left(N_{\mathrm{D}}=3.5 \times 10^{15} \mathrm{~cm}^{-3}\right)$ is used for the experimental study. Before implantation, the wafer is covered with an oxide mask. The $1 \mu \mathrm{m}$ silicon-dioxide $\left(\mathrm{SiO}_{2}\right)$ layer is patterned with a stripe design, see Fig. 1(a). Stripes with a width of $2.2 \mu \mathrm{m}$ and separated from each other by $38 \mu \mathrm{m}$ are etched into the oxide layer. Four regions with different stripe orientations (labeled with $0^{\circ}, 30^{\circ}, 60^{\circ}, 90^{\circ}$ ) are used to screen the implantation such that lateral straggling perpendicular to the stripe opening can be investigated, see Fig. 1(b). The zero-tilt aluminum implantations are performed for different doses and energies at room temperature using a tandem accelerator (NEC, model 5SDH4). The doses are $D=5 \times 10^{13} \mathrm{~cm}^{-2}$ and $D=5 \times 10^{14} \mathrm{~cm}^{-2}$, while the energies are $E=180 \mathrm{keV}$ and $E=280 \mathrm{keV}$. According to SRIM simulations, ${ }^{12}$ the projected ranges of aluminum in $\mathrm{SiC}$ are $200 \mathrm{~nm}$ and $306 \mathrm{~nm}$ for these two energies. After the implantation, the oxide mask is removed, and the samples are measured by SIMS using a CAMECA IMS 7f. An area of $80 \times 80 \mu \mathrm{m}^{2}$ is sputtered by $10 \mathrm{keV} \mathrm{O}{ }_{2}^{+}$ ions. The data detected in image mode are summed up along the particular stripe, i.e., they are projected on the particular plane, in order to increase the counting statistics. Two-dimensional concentration profiles are finally obtained by respective data analysis, where a deconvolution algorithm (Lucy-Richardson) is used to account for the Gaussian distribution of the beam density.

The profile of a high dose $\left(D=5 \times 10^{14} \mathrm{~cm}^{-2}\right)$, low energy $(E=180 \mathrm{keV})$ aluminum implantation is shown in Fig. 2. The stripe pattern used in the investigated region is oriented along [1100]. The implantation is measured from the surface down to a depth of $360 \mathrm{~nm}$. Five distinct regions are marked, which are discussed in the following:

a)

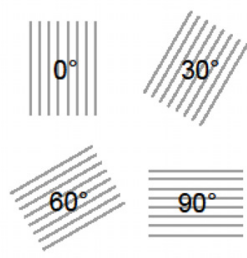

c)

[11̄̄o]

[1रㅓㅇㅣ

[2110]

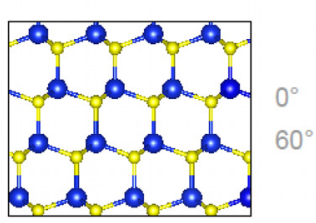

b)

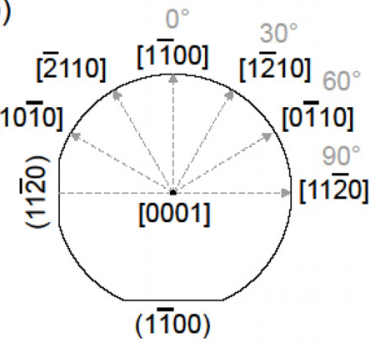

(1100)

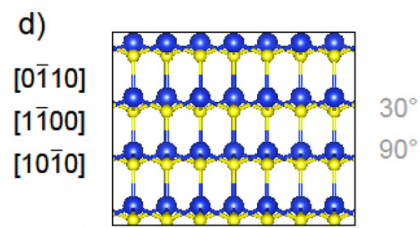

e)

[0001]

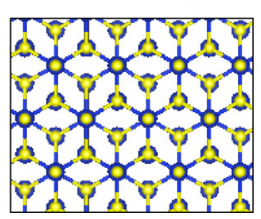

FIG. 1. Implantation mask with its stripes used to guide ions in a particular crystallographic plane (a). Orientation of crystallographic directions of a wafer with major flat $(1 \overline{1} 00)$ (b). Crystal structure of major channeling directions (c)-(e). Silicon atoms are blue, while carbon atoms are yellow.

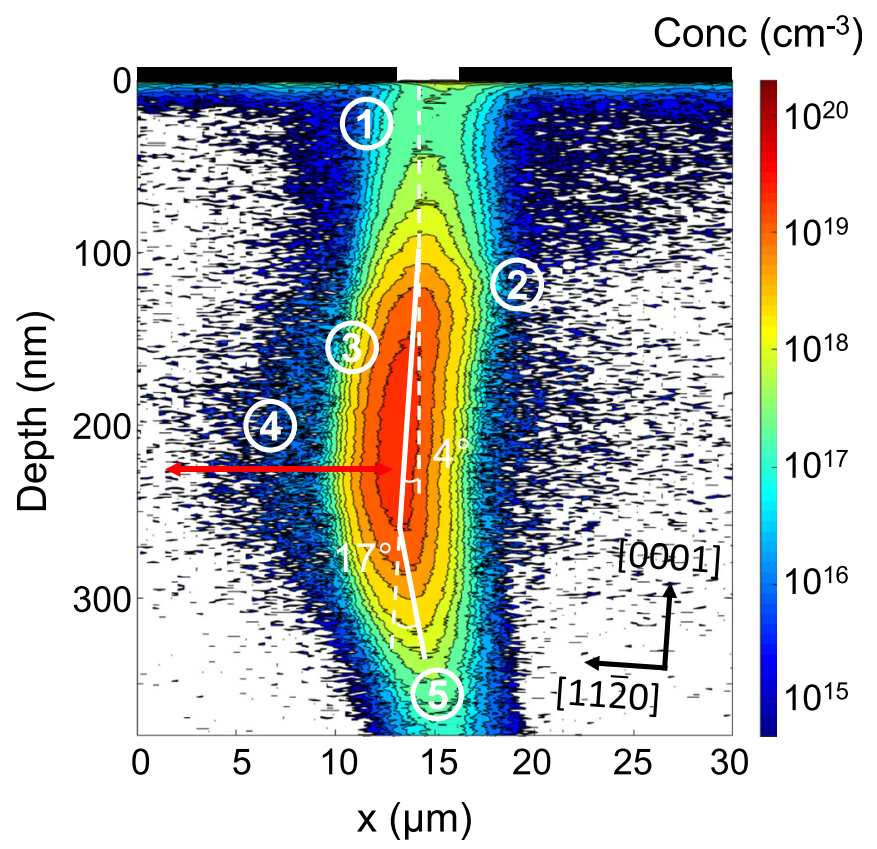

FIG. 2. High resolution profile of an Al implantation with $D=5 \times 10^{14} \mathrm{~cm}^{-2}, E$ $=180 \mathrm{keV}$, and $T_{\text {implant }}=25^{\circ} \mathrm{C}$ from the surface to a depth of $360 \mathrm{~nm}$. The oxide mask is indicated on top of the profile, where the stripe is oriented along [1100]

1. Starting from the surface, most of the penetrating ions follow the surface normal indicated by the dashed line, i.e., they are still following a random trajectory as they have not reached the critical angle for channeling yet. Between the surface and $d \approx 100 \mathrm{~nm}$, the profile is widened in the lateral direction, which is probably caused by a small number of ions scattered at the edges of the oxide mask.

2. When moving further into the epitaxial layer, at $d \approx 110 \mathrm{~nm}$, the profile exhibits a kink on the right side. According to Monte Carlo simulations (Sentaurus Process by Synopsys) using a crystal structure for the target semiconductor, this depth is close to the peak damage $(d \approx 140 \mathrm{~nm})$ generated by the implantation. It appears that the path of the aluminum ions changes here from a random distribution, caused by the strongly damaged environment close to the surface, to a directional propagation defined by the crystalline structure.

3. After the kink, a distinct $4^{\circ}$ tilt of the implantation toward the surface normal can be observed. The tilted path coincides with the [0001] channel of the $4 \mathrm{H}-\mathrm{SiC}$ crystal. A certain amount of aluminum ions is channeled into [0001] instead of following the surface normal. In order to get into this channel, these ions need a reduced energy, probably some $10 \mathrm{keV}$, such that the critical angle becomes larger. ${ }^{14}$ As the penetrating ions lose energy unevenly, they will enter the [0001] channel at different depths, which results in the broadening of a high concentration region to the left of the surface normal.

4. At the projected range, intense lateral spreading of the aluminum ions can be observed. Looking at the minimum detectable doping concentration of $1 \times 10^{15} \mathrm{~cm}^{-3}$, the lateral spread, i.e., the undermask penetration, in $[11 \overline{2} 0]$ is $\sim 10 \mu \mathrm{m}$ (see red arrow). 
Considering the lateral resolution of $2 \mu \mathrm{m}$ of the SIMS beam, the resulting spread is probably less, but still on the order of several micrometers. This lateral spread is substantial and exceeds typical simulation results. Sentaurus Monte Carlo simulations, for example, predict in this case a lateral spread of some $100 \mathrm{~nm}$. However, there is also the literature supporting the extensive lateral spread. Lulli ${ }^{9}$ has found a similar value $(>3 \mu \mathrm{m})$ through Monte Carlo (BCA) simulations. It further corresponds well to the vertical channeling experiments published by Wong-Leung et al.: ${ }^{4}$ for a $60 \mathrm{keV}$ implantation into [1120], the authors found the implantation tail at a depth of $3.5 \mu \mathrm{m}$. Employing these findings toward a $180 \mathrm{keV}$ implantation, the extrapolated depth is in the range of the detected lateral straggling. However, in order to get channeled into $[11 \overline{2} 0]$, the ions need to reach a certain energy to increase the critical angle. ${ }^{14}$ Even though the channel opening is large, see Fig. 1(c), the remaining energy would not be enough to reach large distances. Consequently, we assume that a different mechanism is responsible for the large lateral spread. A similar straggling behavior was previously observed for intrinsic defects in proton-irradiated $4 \mathrm{H}-\mathrm{SiC}$ samples. ${ }^{15}$ These defects are associated with carbon interstitials and are able to move several $100 \mu \mathrm{m}$ in the lateral direction due to recombination processes. In aluminum implanted $\mathrm{SiC}$, defects such as $\mathrm{Al}_{\mathrm{i}}\left(\mathrm{C}_{\mathrm{i}}\right)_{2}$ and $\mathrm{Al}_{\mathrm{Si}+}\left(\mathrm{C}_{\mathrm{i}}\right)_{2}$ can form, ${ }^{16}$ which might obey a similar migration mechanism. These defect complexes appear to be highly stable, also making a long-distance diffusion likely. Further research would be required to estimate the exact equilibrium state and the temperature stability. Furthermore, from the given SIMS results, the distribution of the laterally spread ions appears to be asymmetric. However, it is believed that the straggling is symmetric and that due to the $4^{\circ}$ tilt, the right part of the lateral extension is covered by the nontilted part of the implantation.

5. Further in the $\mathrm{SiC}$ epitaxial layer, the profile tilts back under an angle of $16-18^{\circ}$ with respect to [0001]. The banana-like shape can be explained by ions de-channeling from [0001] due to the high damage around the peak concentration ${ }^{17}$ and subsequently channeling into [11 33 , which is another major channeling direction. ${ }^{4}$ At this depth, the penetrating ions already lost a significant amount of energy, making it more likely to reach the requirements for the critical angle. Compared to the [0001] channel, [11 23$]$ has a larger channeling opening, which increases the penetration range of the implantation tail.

In order to investigate the behavior of other lateral channeling directions, further measurements with differently oriented stripe patterns are performed. For this purpose, the two-dimensional implantation profile is only acquired close to the location of the peak concentration of the implantation, since here, the lateral straggling is most pronounced. First, the region of interest is sputtered down to the peak $(d=315 \mathrm{~nm})$ with a high current beam. Subsequently, a high resolution beam is used to only collect the data for the twodimensional profile. An exemplary implantation with a dose of 5 $\times 10^{13} \mathrm{~cm}^{-2}$, an energy of $280 \mathrm{keV}$, and an implantation temperature of $25^{\circ} \mathrm{C}$ is chosen. Four implantation directions are measured by SIMS: [1 $1 \overline{1} 00],[1 \overline{2} 10],[0 \overline{1} 10]$, and [11 $\overline{2} 0]$. Implanting along [1 $1 \overline{1} 00]$ and $[0 \overline{1} 10]$ leads to lateral channel geometries as depicted in Fig. 1(c). The two other implantation directions, [12 10$]$ and [1120], exhibit a smaller lateral channel opening, see Fig. 1(d). The resulting, $6 \mathrm{~nm}$ deep, two-dimensional profiles taken at the depth of the peak concentration of the implantation are shown in Fig. 3. A lateral spread, i.e., undermask penetration, of around $3-4 \mu \mathrm{m}$ for a concentration of $1 \times 10^{16} \mathrm{~cm}^{-3}$ can be detected for the implantation into [1100] (marked with $0^{\circ}$ ). The same applies for the $60^{\circ}$ implantation, where the ions are implanted along [0110]. Due to the smaller lateral channel, the implantations along [12 10$]\left(30^{\circ}\right)$ and $[11 \overline{2} 0]\left(90^{\circ}\right)$ result in a smaller lateral spread of $2-3 \mu \mathrm{m}$. Figure 4 shows a quantitative overview of the lateral extent of the four profiles, which are summed up over the depth. Comparing the profiles of $0^{\circ}$ and $30^{\circ}$ at $C=1$ $\times 10^{17} \mathrm{~cm}^{-3}$ reveals a difference of $\sim 1 \mu \mathrm{m}$ of the lateral spread. The profiles of $60^{\circ}$ and $90^{\circ}$ show a similar result. The asymmetry of the investigated profiles is most likely caused by the $4^{\circ}$ off-axis growth of the epitaxial layer, resulting in a tilt between the surface normal and the c-axis. Compared to the previous findings (Fig. 2), the amount of lateral straggling is slightly reduced, which can be explained by the one magnitude lower implantation dose and the minimum detectable aluminum concentration of $1 \times 10^{15} \mathrm{~cm}^{-3}$ of the SIMS. Nevertheless, Figs. 3 and 4 reveal significant lateral extensions of the aluminum
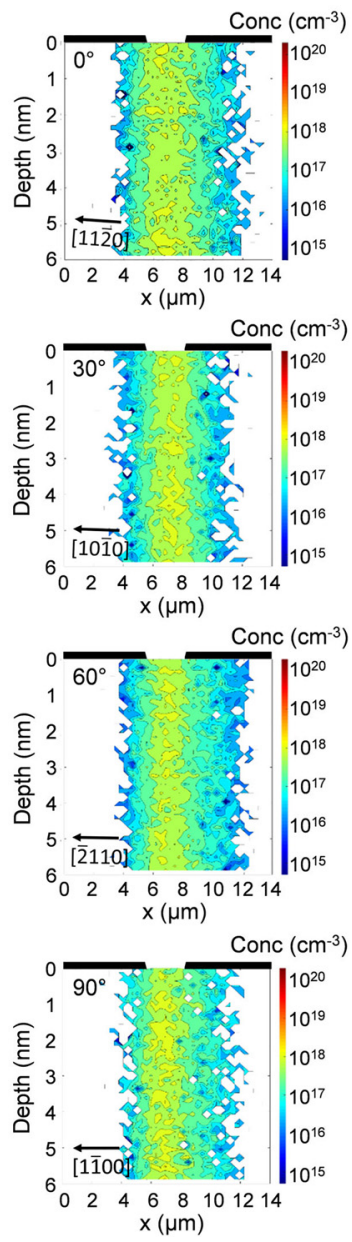

FIG. 3. Al profiles $\left(D=5 \times 10^{13} \mathrm{~cm}^{-2}, E=280 \mathrm{keV}, T=25^{\circ} \mathrm{C}\right)$ of different crystallographic directions. The labeled stripe pattern (e.g., $\left.0^{\circ}\right)$ corresponds to Fig. 1 (b). On top of each profile, the oxide mask is indicated. 


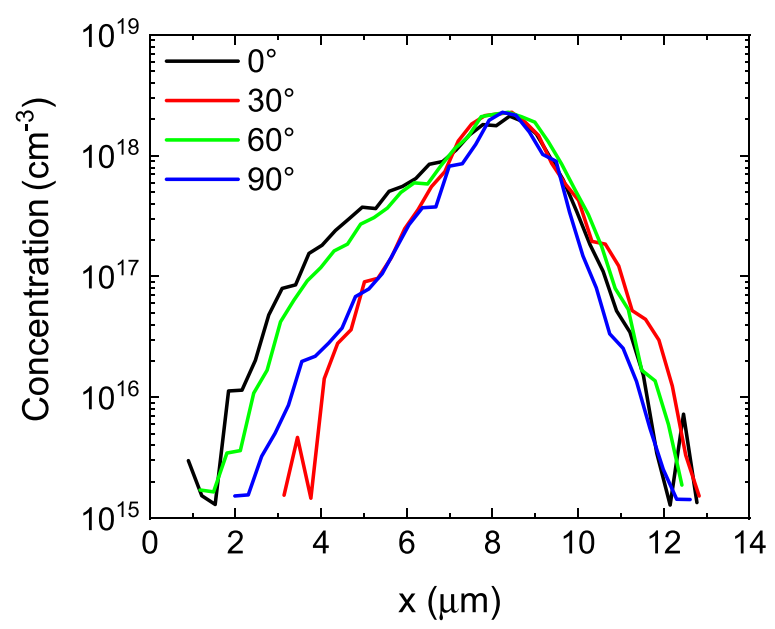

FIG. 4. Profiles shown in Fig. 3 summed up over the depth and aligned with respect to the peak concentration. At $C=1 \times 10^{17} \mathrm{~cm}^{-3}$, the profiles of $0^{\circ}$ and $30^{\circ}$ deviate by $\sim 1 \mu \mathrm{m}$ in the lateral extent.

profile that strongly depend on the particular channeling opening, supporting the previously described two-dimensional profile in Fig. 2.

In summary, aluminum implantations into $4 \mathrm{H}-\mathrm{SiC}$ have been performed at room temperature, and the lateral straggling of different doses and energies has been studied. Using a striped oxide mask, the implantation was screened and different crystallographic directions could be investigated. By using a special approach for SIMS, twodimensional concentration profiles could be acquired. A $360 \mathrm{~nm}$ deep analysis of an aluminum implantation along [1100] has shown a $4^{\circ}$ tilted distribution due to the off axis growth of the epitaxial layer. The significant lateral spread of several micrometers is explained by the large channel opening and supporting migration mechanisms. Comparing aluminum concentration profiles of different directions has shown that lateral straggling highly depends on the corresponding channel opening. These results support the idea that an implantation into $4 \mathrm{H}-\mathrm{SiC}$ can be highly asymmetric and that a significant lateral spread can occur, leading to an unprecise and unreliable doping profile, impacting device behavior.
The Research Council of Norway is acknowledged for the support to the Norwegian Micro- and Nano-Fabrication Facility, NorFab, Project No. 245963.

\section{REFERENCES}

${ }^{7}$ G. Liu, B. R. Tuttle, and S. Dhar, "Silicon carbide: A unique platform for metaloxide-semiconductor physics,” Appl. Phys. Rev. 2, 021307 (2015).

${ }^{2}$ T. Kimoto, "Material science and device physics in $\mathrm{SiC}$ technology for high-voltage power devices,” Jpn. J. Appl. Phys. 54, 040103 (2015).

${ }^{3}$ A. Hallén and M. Linnarsson, "Ion implantation technology for silicon carbide,” Surf. Coat. Technol. 306, 190-193 (2016).

${ }^{4} \mathrm{~J}$. Wong-Leung, M. S. Janson, and B. G. Svensson, "Effect of crystal orientation on the implant profile of $60 \mathrm{keV} \mathrm{Al}$ into $4 \mathrm{H}-\mathrm{SiC}$ crystals," J. Appl. Phys. 93, 8914-8917 (2003).

${ }^{5}$ W. Schustereder, "Challenges for ion implantation in power device processing," ECS Trans. 77, 31-42 (2017).

${ }^{6} \mathrm{~K}$. Mochizuki and N. Yokoyama, "Two-dimensional analytical model for concentration profiles of aluminum implanted into $4 \mathrm{H}-\mathrm{SiC}$ (0001)," IEEE Trans. Electron Devices 58, 455-459 (2011).

${ }^{7}$ E. Morvan, N. Mestres, J. Pascual, D. Flores, M. Vellvehi, and J. Rebollo, "Lateral spread of implanted ion distributions in 6H-SiC: Simulation," Mater. Sci. Eng., B 61-62, 373-377 (1999).

${ }^{8}$ T. B. Hook, J. Brown, P. Cottrell, E. Adler, D. Hoyniak, J. Johnson, and R. Mann, "Lateral ion implant straggle and mask proximity effect," IEEE Trans. Electron Devices 50, 1946-1951 (2003).

${ }^{9} \mathrm{G}$. Lulli, "Two-dimensional simulation of undermask penetration in $4 \mathrm{H}-\mathrm{SiC}$ Implanted with Al+ ions," IEEE Trans. Electron Devices 58, 190-194 (2011).

${ }^{10} \mathrm{R}$. Elpelt, B. Zippelius, S. Doering, and U. Winkler, "Employing scanning spreading resistance microscopy (SSRM) for improving TCAD simulation accuracy of silicon carbide," Mater. Sci. Forum 897, 295-298 (2017).

${ }^{11}$ V. Raineri, V. Privitera, and E. Rimini, "Lateral straggling of B and P ions implanted in channeling and random directions of Si single crystals," Appl. Phys. Lett. 61, 1190-1192 (1992).

${ }^{12}$ J. F. Ziegler, see http://www.srim.org/ for "SRIM."

${ }^{13}$ D. S. C. Biggs and M. Andrews, "Image restoration algorithms," Appl. Opt. 36, 1766-1775 (1997).

${ }^{14} \mathrm{G}$. Hobler, "Critical angles and low-energy limits to ion channeling in silicon," Radiat. Eff. Defects Solids 139, 21-85 (1996).

${ }^{15}$ L. S. Løvlie, L. Vines, and B. G. Svensson, "A Laterally resolved DLTS study of intrinsic defect diffusion in $4 \mathrm{H}-\mathrm{SiC}$ after low energy focused proton beam irradiation," Mater. Sci. Forum 645-648, 431-434 (2010).

${ }^{16}$ T. Hornos, A. Gali, N. T. Son, and E. Janzén, "A theoretical study on aluminiumrelated defects in SiC,” Mater. Sci. Forum 556-557, 445-448 (2007).

${ }^{17}$ N. G. Chechenin, K. K. Bourdelle, A. V. Suvorov, and A. X. Kastilio-Vitloch, "Damage and aluminum distributions in sic during ion implantation and annealing," Nucl. Instrum. Methods Phys. Res., B 65, 341-344 (1992). 